\title{
Transient Airflow Process Across three Vertical Vents Induced by Stack- Driven Effect Inside Un- Stratified Cross- Ventilated Rectangular Building with an Opposing Flow in One of the Upper Opening
}

\author{
A. L. Muhammad \\ Department of Mathematics \\ Kano University of Science \\ and Technology \\ Gaya Road- Wudil PMB 3244
}

\author{
A. B. Baffa \\ Department of Computer \\ Sciences \\ Bayero University Kano \\ Gwarzo Road PMB 3011
}

\author{
U. M. Dauda \\ Department of Mathematics \\ Kano University of Science \\ and Technology \\ Gaya Road- Wudil PMB 3244
}

\begin{abstract}
In the present study, an analytical solution for transient airflow process across three vertical vents induced by stackdriven effect in un- stratified cross- ventilated rectangular building with an opposing flow in one of the upper opening was presented. An approximation of reduced gravity is taken into account in order to maintained thermal buoyancy effect. One dimensional Navier- Stokes Equations is utilized to model the airflow process in the building. Variation of parameter and separation of variable methods were employed to obtain the possible solutions of the model equations. The solutions predicts the following; velocity- and temperature profiles together with volumetric airflow and mass transfer which evaluated numerically for several sets of values of effective thermal coefficient $\left(\theta_{0}\right)$.
\end{abstract}

\section{Keywords}

Velocity profiles; Temperature profiles; volumetric airflow; Mass transfer

\section{INTRODUCTION}

Generally, Natural ventilation of building provides improvement of internal comfort and air quality conditions leading to a significant reduction of cooling energy consumption. Design of natural ventilation systems for many types of building is based on buoyancy forces. However, external wind flow can have significant effects on stackdriven natural ventilation. Air flow distributions in buildings are considered to be as a result of the knowledge of the exact air supply to a building. This is necessary to determine its thermal performance and the concentration of the indoor pollutants. The exchange of air can be achieved either by mechanical means (Mechanical ventilation) or through the large opening of the building envelope (Natural ventilation). Exchanges between external ambience and interior space of buildings caused by flows that are driven by wind or by temperature differences are the foundation of natural ventilation process. However, natural ventilation is being pursued by humans, who are increasingly spending more time indoors, to extend the possibilities of living in uncongenial or squally conditions etc. The improvements of the quality of the interior space both in its attractiveness, spaciousness, luminosity, and more importantly its proper natural ventilation are major concerns for designers of modern structures. Air flow modeling gives Architectures and Engineers the luxury to consider several design options in the minimum amount of time. As a result, the final design is not based on a tentative approach but on a professional design process considering several options and selecting the best design. Air flow models are used to simulate the rates of incoming and outgoing air flows for a domain with known leakages under given weather and shielding conditions. Air flow models can be divided into two main categories, single-zone models and multi-zone models. Single-zone models assume that the structure can be described by a single, well mixed zone. Many attempts to investigate this phenomenon have been made by some researchers. [4] Studied a simple basic theory of natural convection across openings in vertical partitions and generalized to include both heat and mass-transfer in a singlesided ventilated domain. Displacement ventilation (where the interior is stratified) was studied by [7], and the mixing ventilation (where the interior has uniform temperature) by [2]. [1] Studied a convective heat and mass-transfer through large openings, which plays an important role in the thermal behavior of domains. [5] Considered building having two openings at different vertical level on opposite walls, the heights of the two openings are relatively small, and the areas of the top and bottom openings are $A_{t}$ and $A_{b}$ respectively. The study also considered an indoor source of heat $E$, and the wind force can assist or oppose the thermal buoyancy force, when the indoor temperature is uniform. [3] Considered natural ventilation in a full-scale building induced by combined wind and buoyancy forces. The overall objectives were to verify and validate a CFD model for the naturally ventilated buildings, collect high quality full-scale experimental data for CFD validation and formulate guidelines for modeling natural ventilation in design practice and a steady envelope flow model were applied to calculate mean ventilation rates. [6] Investigated the study on combination of natural ventilation methods. A test room of single-sided ventilation was equipped with a vertical vent. Ventilation rate through the openings was evaluated based on the air flow velocity measured at the surface area of the openings. The vertical vent was kept closed during the first run of the experiments then the same experiments repeated where the vent was in use. Based on the experimental results, the effects of the vertical vent on the ventilation rate were clarified and a model was suggested based on combination for the two ventilation methods. [8] Investigated air flow rate across a vertical opening induced by a thermal source in a room, various parameters were used in designing natural ventilation. [9] Considered wind-driven cross ventilation in building with small openings. [11] Studied airflow process across vertical vents induced by stack- driven effect with an opposing flow in one of the openings was presented. An investigation of stack- driven airflow through rectangular 
cross- ventilated building with two openings using analytic technique was also recently presented by [12]. [10] Presented a simple mathematical model of stack ventilation flows in multi-compartment buildings, with a view to providing an intuitive understanding of the physical processes governing the movement of air and heat through naturally ventilated buildings. In which, the solution for the velocity, temperature distributions and volumetric airflow, mass- transfer rates were obtained.

The main objective of this paper is to analytically determine the temperature-, velocity profiles together with volumetric airflow and mass transfer in a rectangular un- stratified crossventilated building with three openings on a vertical wall. Analytical results for the temperature-, velocity profiles together with volumetric airflow and mass transfer are presented and discussed graphically. This is the novel approach which will lead to better understanding of the phenomenon and help in optimizing the designs for better natural ventilation.

\section{DOMAIN DESCRIPTION}

The building considered, is un-stratified cross- ventilated rectangular building with three openings. In which the building has two upper and one lower rectangular opening. The two upper openings have an area of $0.7 \mathrm{~m} \times 1.0 \mathrm{~m}$, while the one lower opening is $0.7 \mathrm{~m} \times 2.0 \mathrm{~m}$. Dimension of the building is $5.3 \mathrm{~m} \times 3.6 \mathrm{~m} \times 2.8 \mathrm{~m}$ with air as the connecting fluid. The domain envelops were separated from one another by a vertical rectangular openings of height $y^{*}$ and width $x_{w}$, which is illustrated in Figure 1. The density of air in the building is maintained at $\rho_{0}$ with temperature at $\dot{\theta}^{*}$ and pressure $P$.

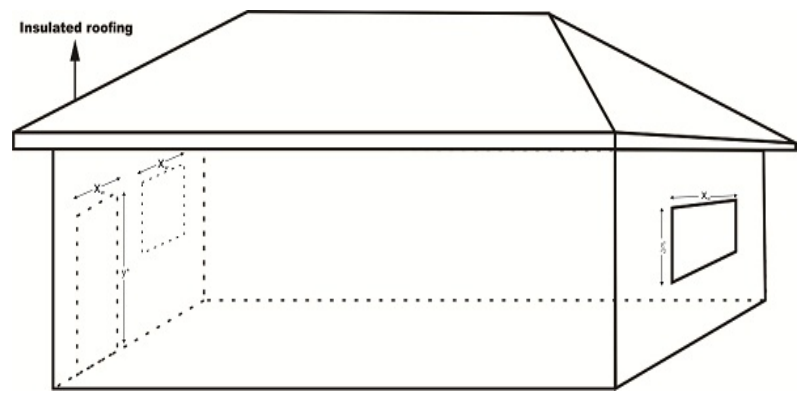

Figure 1: Diagram of un-stratified cross ventilated rectangular building with three openings.

\section{MODEL FORMULATION}

In the present paper we discussed the temperature- velocityprofiles together with volumetric and mass transfer for transient Stack- driven airflow through rectangular openings in building with three openings in the presence of uniform interior temperature (see Figure 1). Schematic diagrams of airflow process inside the building and the one vertical upper vent are shown in Figure 2 and 3 below. The flow is transient that depends on the height of the opening on the vertical walls. Airflow is assumed to be at low speed so that it will behave like incompressible fluid. Internal heat source is negligible $q \ll 1$ (see Figure 2 and 3). An approximation of reduced gravity is invoked. One Navier Stokes Equations with appropriate boundary conditions will describe the problem. The model equations are written in a dimensionless form and solved analytically by means of separation of variable methods.

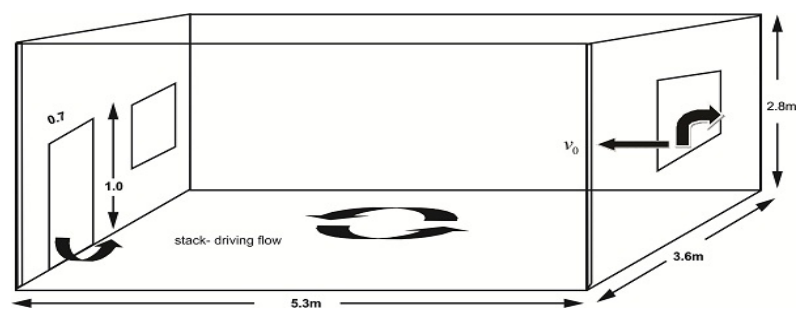

Figure 2 Schematic diagram of airflow process inside unstratified cross ventilated rectangular building with three openings.

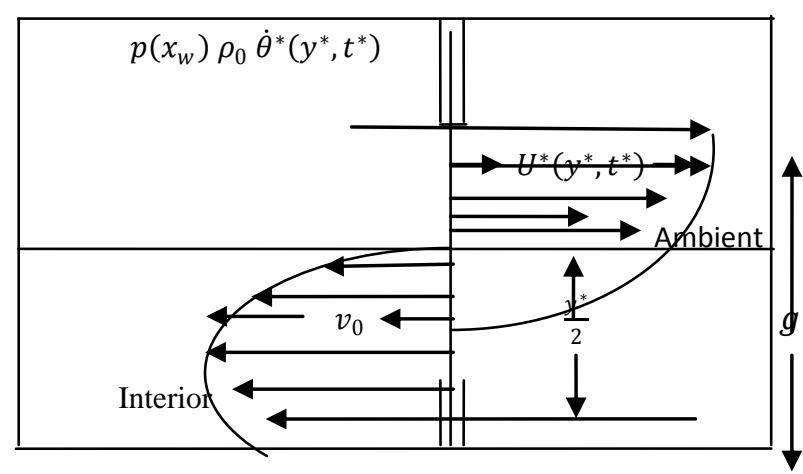

Figure 3 Schematic diagram of airflow process across one of the vertical upper vent with an opposing flow in rectangular building with three openings.

The convective motion induced by stack- driven effect as illustrated in Figure 2 and 3 is described by the conservation Equations for continuity, momentum and energy Equations given by,

$$
\begin{aligned}
& \rho_{0}\left(\frac{\partial u}{\partial x_{w}}+\frac{\partial v}{\partial y}\right)=0 \\
& \rho_{0}\left(\frac{\partial u}{\partial t}+u \frac{\partial u}{\partial x_{w}}+v \frac{\partial v}{\partial y}\right)=-\frac{\partial p}{\partial x_{w}}+\mu\left(\frac{\partial^{2} u}{\partial x_{w}{ }^{2}}+\frac{\partial^{2} u}{\partial y^{2}}\right) \\
& \rho_{0}\left(\frac{\partial \theta}{\partial t}+u \frac{\partial \theta}{\partial x_{w}}+v \frac{\partial \theta}{\partial y}\right)=\frac{\kappa}{c_{p}}\left(\frac{\partial^{2} \theta}{\partial x_{w}{ }^{2}}+\frac{\partial^{2} \theta}{\partial y^{2}}\right)+\frac{q}{c_{p}} \phi_{v}
\end{aligned}
$$

It assumed that the velocity and temperature fields are independent of the distance parallel to the surface, and if the gravitational field is aligned with the direction of air motion the pressure will be a component along the width of the opening in the building, and air as a non viscous fluid we can neglecting viscous dissipation as $\Phi_{v} \ll 1$, and $q \ll 1$. So we have,

$U=u(y, t), v=v_{0}=$ const. $\dot{\theta}=\theta(y, t), P=p\left(x_{w}\right)$

Navier-Stokes Equations are simplified by the above mentioned assumptions in E. In which Continuity Equation is satisfied identically then, Equations (2) and (3) can be reduced to one dimensional momentum and Energy Equation that describes the problems as,

$\frac{\partial U}{\partial t}+v_{0} \frac{\partial U}{\partial y}=g \beta \Delta \dot{\theta}+v \frac{\partial^{2} U}{\partial y^{2}}$.

$\frac{\partial \dot{\theta}}{\partial t}+v_{0} \frac{\partial \dot{\theta}}{\partial y}=\alpha \frac{\partial^{2} \dot{\theta}}{\partial y^{2}}$.

With the following dimensional boundary conditions as,

$0 \leq y \leq 2, t \geq 0, U(0)=0, U(2)=0, U(0, t)=0$, $U(2, t)=0, \theta(0)=-\theta_{0}, \theta(2)=1-\theta_{0}, \theta(0, t)=$ $0, \theta(2, t)=0$. 
By scaling $y$ with $y^{*} L$, velocity $U$ with $\frac{U^{*} g \beta \Delta \theta L^{2}}{\alpha}, v_{0}=\frac{v_{0}{ }^{*} v}{L}$, $t=\frac{t^{*} L^{2}}{\alpha}$, and introducing $\dot{\theta}$ with $\dot{\theta}^{*} \Delta \theta+\theta_{0}$, where $\Delta \dot{\theta}=\dot{\theta}-$ $\theta_{0}$.

In dimensionless form the above Equations (5) and (6) may be expressed as,

$\frac{\partial U^{*}}{\partial t^{*}}-C \frac{\partial U^{*}}{\partial y^{*}}=\operatorname{Pr} \frac{\partial^{2} U^{*}}{\partial y^{* 2}}+\dot{\theta}^{*}\left(y^{*}, t\right)$

$\frac{\partial \dot{\theta}^{*}}{\partial t^{*}}-C \frac{\partial \dot{\theta}^{*}}{\partial y^{*}}=\frac{\partial^{2} \dot{\theta}^{*}}{\partial y^{* 2}}$

Where, $-C=v_{0} P r$.

With the following dimensionless boundary conditions as,

$0 \leq y^{*} \leq 1, t^{*} \geq 0, U^{*}(0)=0, U^{*}(1)=0, U_{u}^{*}\left(0, t^{*}\right)=0$,

$U_{u}^{*}\left(1, t^{*}\right)=0, \frac{\partial U^{*}\left(1, t_{\max }\right)}{\partial y^{*}}=U_{0}, \dot{\theta}^{*}(0)=-\theta_{0}, \dot{\theta}^{*}(1)=1-$ $\theta_{0}, \dot{\theta}_{u}^{*}\left(0, t^{*}\right)=0, \dot{\theta}_{u}^{*}\left(1, t^{*}\right)=0, \dot{\theta}_{u}^{*}\left(1, t_{\max }\right)=\operatorname{Sin} t^{*}$.

\section{SOLUTION OF THE DIMENSIONLESS MODEL EQUATIONS}

\subsection{Dimensionless temperature profiles} Steady solution for dimensionless temperature profiles

The steady state Equation and boundary condition for dimensionless temperature profiles is,

$\frac{d^{2} \dot{\theta}^{*}}{d y^{* 2}}+C \frac{d \dot{\theta}^{*}}{d y^{*}}=0$

$0 \leq y^{*} \leq 1, \dot{\theta}^{*}(0)=-\theta_{0}, \dot{\theta}^{*}(1)=1-\theta_{0}$

The resulting solution is,

$\dot{\theta}_{s}^{*}\left(y^{*}\right)=-\theta_{0}+\frac{e^{C}}{1-e^{C}}\left(e^{-C y}-1\right)$

Time dependent solution for dimensionless temperature profiles

The Equation for the temperature profiles is one given in Equation (8) as,

$\frac{\partial \dot{\theta}^{*}}{\partial t^{*}}-C \frac{\partial \dot{\theta}^{*}}{\partial y^{*}}=\frac{\partial^{2} \dot{\theta}^{*}}{\partial y^{* 2}}$

The separation between the steady and unsteady part of solution are as follows,

$\dot{\theta}^{*}\left(y^{*}, t\right)=\dot{\theta}^{*}{ }_{s}\left(y^{*}\right)+\dot{\theta}_{u}^{*}\left(y^{*}, t^{*}\right)$

The Equation (8) is also valid for the unsteady part of the solution as,

$$
\frac{\partial \dot{\theta}_{u}^{*}}{\partial t^{*}}-C \frac{\partial \dot{\theta}_{u}^{*}}{\partial y^{*}}=\frac{\partial^{2} \dot{\theta}_{u}^{*}}{\partial y^{* 2}}
$$

With the following boundary condition for dimensionless temperature profiles as,

$0 \leq y^{*} \leq 1, \dot{\theta}_{u}^{*}\left(0, t^{*}\right)=0, \dot{\theta}_{u}^{*}\left(1, t^{*}\right)=0, \dot{\theta}_{u}^{*}\left(1, t_{\max }\right)=$ Sint*.

The separation given by,

$\dot{\theta}_{u}^{*}\left(y^{*}, t^{*}\right)=Y\left(y^{*}\right) T\left(t^{*}\right)$

Leads with Equation (12) to the eigen value problem as,

$\frac{T^{\prime}}{T}=\frac{Y^{\prime \prime}}{Y}+\frac{C Y^{\prime}}{Y}=-P_{1}^{2}$ for $P_{1}>0$.

With generalized solution of the form, $\dot{\theta}_{u}^{*}\left(y^{*}, t^{*}\right)=e^{-\left(P_{1}^{2} t^{*}+\frac{c}{2}\right)}\left(K_{1} \cosh ^{*}+K_{2} \sinh ^{*}\right)$

Equation (15), together with the homogeneous dimensionless boundary conditions yields to,

$\dot{\theta}_{u}^{*}\left(y^{*}, t^{*}\right)=K_{2} e^{-\left(P_{1}^{2} t^{*}+\frac{C}{2}\right)} \operatorname{coshay^{*}}$

Where, $K_{1}=0, K_{2}=\frac{\sin t^{*}}{\cosh a} e^{P_{1}^{2} t_{\max }+\frac{C}{2}}, a=\frac{\sqrt{C^{2}-4 P_{1}^{2}}}{2}$ at $\dot{\theta}_{u}^{*}\left(1, t_{\max }\right)=\operatorname{Sint}^{*}, t^{*} \geq 0$.

The resulting Equation (16) becomes,

$\dot{\theta}_{u}^{*}\left(y^{*}, t^{*}\right)=\frac{\sin t^{*}}{\cosh a} e^{P_{1}^{2}\left(t_{\max }-t^{*}\right)} \cosh a y^{*}$

The general time dependent solution for dimensionless temperature profiles is,

$\dot{\theta}^{*}\left(y^{*}, t^{*}\right)=$
$-\theta_{0}+\frac{e^{C}}{1-e^{C}}\left(e^{-C y^{*}}-1\right)+\frac{\sin t^{*}}{\cosh a} e^{P_{1}^{2}\left(t_{\max }-t^{*}\right)} \cosh a y^{*}$.

\subsection{Dimensionless velocity profiles}

Steady state solution for dimensionless velocity profiles

The steady state Equation and boundary condition for dimensionless velocity profiles is,

$-C \frac{d U^{*}}{d y^{*}}=\operatorname{Pr} \frac{d^{2} U^{*}}{d y^{* 2}}+\dot{\theta}^{*}\left(y^{*}\right)$

$0 \leq y^{*} \leq 1, U^{*}(0)=0, U^{*}(1)=0$.

Plugging the Equation (10) in Equation (19) yields to,

$-C \frac{d U^{*}}{d y^{*}}=\operatorname{Pr} \frac{d^{2} U^{*}}{d y^{* 2}}-\theta_{0}+\frac{e^{C}}{1-e^{C}}\left(e^{-C y^{*}}-1\right)$.

Starting with the homogeneous part of Equation (19), one obtained the complementary solution as,

$U_{c}\left(y^{*}\right)=C_{1}+C_{2} e^{v_{0} y^{*}}$.

By employing the variation of parameter methods, one can write the particular solution as,

$U_{P}\left(y^{*}\right)=\frac{1}{C^{2}\left(1-e^{-C}\right)}\left[\left(\theta_{0}\left(1-e^{-C}\right)-1\right) C y^{*}-e^{-C y^{*}}(1+\right.$

$\left.\left.\frac{P r}{1-P r}\right)+\operatorname{Pr}\left(1-\theta_{0}\left(1-e^{-C}\right)\right)\right]$.

The general solution is given by,

$U_{s}^{*}\left(y^{*}\right)=C_{1}+C_{2} e^{v_{0} y^{*}}+\frac{1}{C^{2}\left(1-e^{-C}\right)}\left[\left(\theta_{0}\left(1-e^{-C}\right)-\right.\right.$

1) $\left.C y^{*}-e^{-C y^{*}}\left(1+\frac{P r}{1-P r}\right)+\operatorname{Pr}\left(1-\theta_{0}\left(1-e^{-C}\right)\right)\right]$

The resulting solution for steady dimensionless velocity profiles is,

$U_{S}^{*}\left(y^{*}\right)=\frac{1}{C^{2}\left(1-e^{-C}\right)\left(1-e^{\left.v_{0}\right)}\right.}\left[\left(1+\frac{P r}{1-P r}\right)\left(e^{-C}-e^{v_{0}}-\right.\right.$

$\left.e^{v_{0} y^{*}}\left(e^{-C}-1\right)-e^{-C y^{*}}\left(1-e^{v_{0}}\right)\right)+\left(1-\theta_{0}(1-\right.$

$\left.\left.\left.e^{-C}\right)\right)\left(C-\operatorname{Pr}\left(1-e^{v_{0}}\right)-C e^{v_{0} y^{*}}+\left(1-e^{v_{0}}\right)\left(\operatorname{Pr}-C y^{*}\right)\right)\right]$.

Where, $C_{1}=$

$\frac{\left(1+\frac{P r}{1-P r}\right)\left(e^{-C}-e^{-\frac{C}{P r}}\right)+\left(1-\theta_{0}\left(1-e^{-C}\right)\right)\left(C-P r\left(1-e^{-\frac{C}{P r}}\right)\right)}{C^{2}\left(1-e^{-C}\right)\left(1-e^{-\frac{C}{P r}}\right)}, C_{2}=$
$-\frac{1}{C^{2}\left(1-e^{-C}\right)\left(1-e^{-\frac{C}{P r}}\right)}\left[\left(1+\frac{P r}{1-P r}\right)\left(e^{-C}-1\right)+C(1-\right.$
$\left.\left.\theta_{0}\left(1-e^{-C}\right)\right)\right]$ 
Time- dependent solution for dimensionless velocity profiles

Plugging the Equation (18) in Equation (7) yields to,

$\frac{\partial U^{*}}{\partial t^{*}}-C \frac{\partial U^{*}}{\partial y^{*}}=\operatorname{Pr} \frac{\partial^{2} U^{*}}{\partial y^{* 2}}-\theta_{0}+\frac{e^{C}}{1-e^{C}}\left(e^{-C y^{*}}-1\right)+$

$\frac{\sin t^{*}}{\cosh a} e^{P_{1}^{2}\left(t_{\max }-t^{*}\right)} \cosh a y^{*}$.

The separation between the steady and unsteady part of solution are as follows,

$U^{*}\left(y^{*}, t^{*}\right)=U_{s}^{*}\left(y^{*}\right)+U_{u}^{*}\left(y^{*}, t^{*}\right)$

The Equation (25) is also valid for the unsteady part of the solution as,

$\frac{\partial U_{u}^{*}}{\partial t^{*}}-C \frac{\partial U_{u}^{*}}{\partial y^{*}}=\operatorname{Pr} \frac{\partial^{2} U_{u}^{*}}{\partial y^{* 2}}-\theta_{0}+\frac{e^{C}}{1-e^{C}}\left(e^{-C y^{*}}-1\right)+$

$\frac{\sin t^{*}}{\cosh a} e^{P_{1}^{2}\left(t_{\max }-t^{*}\right)} \cosh a y^{*}$.

With the following boundary condition for velocity profiles as,

$0 \leq y^{*} \leq 1, U_{u}^{*}\left(0, t^{*}\right)=0, U_{u}^{*}\left(1, t^{*}\right)=0, \frac{\partial U^{*}\left(1, t_{\max }\right)}{\partial y^{*}}=U_{0}$.

Starting with the homogeneous part of Equation (25), one obtain

$\frac{d U^{*}}{d t^{*}}-C \frac{d U^{*}}{d y^{*}}-\operatorname{Pr} \frac{d^{2} U^{*}}{d y^{* 2}}=0$.

The separation is given by the complementary solution as,

$U_{c}^{*}\left(y^{*}, t^{*}\right)=Y\left(y^{*}\right) T\left(t^{*}\right)$

Leads with Equation (28) to the eigen value problem as,

$\frac{T^{\prime}}{T}=\operatorname{Pr} \frac{Y^{\prime \prime}}{Y}+\frac{C Y^{\prime}}{Y}=-P_{1}^{2}$ for $P_{1}>0$.

The generalized complementary solution is of the form,

$U_{C}^{*}\left(y^{*}, t^{*}\right)=e^{-\left(P_{1}^{2} t^{*}-\frac{1}{2} v_{0} y^{*}\right)}\left(C_{3} \cosh K y^{*}+C_{4} \sinh K y^{*}\right)$

Using the boundary condition for unsteady velocity profiles, one obtain,

$U_{C}^{*}\left(y^{*}, t\right)=\frac{U_{0} \sinh K y^{*} e^{P_{1}^{2}\left(\left(t_{\max }-t^{*}\right)-\frac{1}{2} v_{0}\left(1-y^{*}\right)\right)}}{K \cosh K-\frac{C}{2 P r} \sin K}$

Where, $C_{3}=0, C_{4}=\frac{U_{0} e^{\left(P_{1}^{2} t_{\max }-\frac{1}{2} v_{0}\right)}}{K \cos h K-\frac{C}{2 \operatorname{Pr}} \sin K}, K=\frac{\sqrt{C^{2}-4 \operatorname{Pr} P_{1}^{2}}}{2 \operatorname{Pr}}$.

The particular solution for Equation (25) is given by,

$$
\begin{gathered}
U_{P}^{*}\left(y^{*}, t^{*}\right)=\frac{-e^{P_{1}^{2}\left(t_{\max }-t^{*}\right)} \sin t^{*}}{2 \cosh a}\left(\frac{e^{a y^{*}}}{1+P_{1}^{2}+a c}+\frac{e^{-a y^{*}}}{1+P_{1}^{2}-a c}\right)- \\
\theta_{0} t^{*}+C \theta_{0} y^{*}+\frac{P r y^{* 2} \theta_{0}}{2}+\frac{e^{C}}{\left(1-e^{C}\right)}\left(t^{*}-C y^{*}-\frac{P r y^{* 2}}{2}\right)
\end{gathered}
$$

The generalized solution of unsteady velocity profiles is of the form,

$U_{u}^{*}\left(y^{*}, t^{*}\right)=U_{C}^{*}\left(y^{*}, t^{*}\right)+U_{P}^{*}\left(y^{*}, t^{*}\right)$

This yield to,

$U_{u}^{*}\left(y^{*}, t^{*}\right)=$

$\frac{U_{0} \sinh K y^{*} e^{P_{1}^{2}\left(\left(t_{\max }-t^{*}\right)-\frac{1}{2} v_{0}\left(1-y^{*}\right)\right)}}{K \cosh K-\frac{C}{2 P r} \sin K}-\frac{-e^{P_{1}^{2}\left(t_{\max }-t^{*}\right)} \sin t^{*}}{2 \cosh a}\left(\frac{e^{a y^{*}}}{1+P_{1}^{2}+a c}+\right.$

$\left.\frac{e^{-a y^{*}}}{1+P_{1}^{2}-a c}\right)-\theta_{0} t^{*}+C \theta_{0} y^{*}+\frac{\operatorname{Pr}^{* 2} \theta_{0}}{2}+\frac{e^{C}}{\left(1-e^{C}\right)}\left(t^{*}-C y^{*}-\right.$

$\left.\frac{\operatorname{Pr} y^{* 2}}{2}\right)$
Therefore, the general time dependent solution for dimensionless velocity profiles given in Equation (27) yields to,

$$
\begin{aligned}
& U^{*}\left(y^{*}, t^{*}\right)=\frac{1}{C^{2}\left(1-e^{-C}\right)\left(1-e^{v_{0}}\right)}\left[( 1 + \frac { P r } { 1 - P r } ) \left(e^{-C}-e^{v_{0}}-\right.\right. \\
& \left.e^{v_{0} y^{*}}\left(e^{-C}-1\right)-e^{-C y^{*}}\left(1-e^{v_{0}}\right)\right)+\left(1-\theta_{0}(1-\right. \\
& \left.\left.e^{-C}\right)\right)\left(C-\operatorname{Pr}\left(1-e^{v_{0}}\right)-C e^{v_{0} y^{*}}+\left(1-e^{v_{0}}\right)(P r-\right. \\
& \left.\left.\left.C y^{*}\right)\right)\right]+\frac{\left.U_{0} \sinh K y^{*} e^{P_{1}^{2}\left(\left(t_{\max }-t^{*}\right)-\frac{1}{2} v_{0}\left(1-y^{*}\right)\right.}\right)}{K \cosh K-\frac{C}{2 P r} \sin K}- \\
& \frac{e^{P_{1}^{2}\left(t_{\max }-t^{*}\right)}}{2 \cosh a} \sin t^{*}\left(\frac{e^{a y^{*}}}{1+P_{1}^{2}+a c}+\frac{e^{-a y^{*}}}{1+P_{1}^{2}-a c}\right)-\theta_{0} t^{*}+C \theta_{0} y^{*}+ \\
& \frac{\operatorname{Pr} y^{* 2} \theta_{0}}{2}+\frac{e^{C}}{\left(1-e^{C}\right)}\left(t^{*}-C y^{*}-\frac{\operatorname{Pr} y^{* 2}}{2}\right) .
\end{aligned}
$$

\subsection{Dimensionless volumetric airflow}

The volumetric airflow is defined in Equation (36) below,

$Q^{*}\left(y^{*}, t^{*}\right)=A^{*} c_{d} \iint_{s=0}^{s=\frac{y^{*}}{2}} U^{*}(s) d s d t^{*}$.

Putting Equation (35) in (36), one obtains

$$
\begin{aligned}
& Q^{*}\left(y^{*}, t^{*}\right)=A^{*} c_{d} \iint_{s=0}^{s=\frac{y^{*}}{2}}\left[\frac{1}{C^{2}\left(1-e^{-C}\right)\left(1-e^{v_{0}}\right)}[(1+\right. \\
& \left.\frac{P r}{1-P r}\right)\left(e^{-C}-e^{v_{0}}-e^{v_{0} s}\left(e^{-C}-1\right)-e^{-C s}\left(1-e^{v_{0}}\right)\right)+ \\
& \left(1-\theta_{0}\left(1-e^{-C}\right)\right)\left(C-\operatorname{Pr}\left(1-e^{v_{0}}\right)-C e^{v_{0} s}+\right. \\
& \left.\left.\left(1-e^{v_{0}}\right)(\operatorname{Pr}-C s)\right)\right]+\frac{\left.U_{0} \sinh K s^{*} e^{P_{1}^{2}\left(\left(t_{\max }-t^{*}\right)-\frac{1}{2} v_{0}(1-s)\right.}\right)}{K \cosh K-\frac{C}{2 P r} \sin K}- \\
& \frac{e^{P}{ }_{1}^{2}\left(t_{\max }-t^{*}\right)}{2 \cos h a} \sin t^{*}\left(\frac{e^{a s}}{1+P_{1}^{2}+a c}+\frac{e^{-a s}}{1+P_{1}^{2}-a c}\right)-\theta_{0} t^{*}+C \theta_{0} s+ \\
& \left.\frac{\operatorname{Prs}^{2} \theta_{0}}{2}+\frac{e^{C}}{\left(1-e^{C}\right)}\left(t^{*}-C s-\frac{\operatorname{Prs}^{2}}{2}\right)\right] d s d t^{*} .
\end{aligned}
$$

Where, $s$ is a dummy variable.

One obtain the volumetric airflow as,

$$
\begin{aligned}
& Q^{*}\left(y^{*}, t^{*}\right)=A^{*} c_{d}\left[\frac { 1 } { c ^ { 2 } ( 1 - e ^ { - C } ) ( 1 - e ^ { v 0 } ) } \left(( 1 + \frac { P r } { 1 - P r } ) \left(\left(e^{-C}-\right.\right.\right.\right. \\
& e^{\left.v_{0}\right) \frac{y^{*}}{2}}-\left(e^{-C}-1\right) \frac{e^{v_{0} \frac{y^{*}}{2}}}{v_{0}}+\left(1-e^{v_{0}}\right) \frac{e^{-C \frac{y^{*}}{2}}}{C}+\frac{\left(e^{-C}-1\right)}{v_{0}}- \\
& \left.\frac{\left(1-e^{v_{0}}\right)}{C}\right)+\left(1-\theta_{0}\left(1-e^{-C}\right)\right)\left(-\operatorname{Pr}\left(v_{0}+\left(1-e^{v_{0}}\right)\right) \frac{y^{*}}{2}+\right.
\end{aligned}
$$$$
\left.\left.\operatorname{Pr} e^{v_{0} \frac{y^{*}}{2}}+\left(1-e^{v_{0}}\right)\left(\operatorname{Pr} \frac{y^{*}}{2}-C \frac{y^{* 2}}{8}\right)-\operatorname{Pr}\right)\right) t^{*}+
$$$$
\frac{U_{0} \frac{v_{0}}{2} e^{P_{1}^{2}\left(\left(t_{\max }-t^{*}\right)+\frac{1}{2} v_{0}\right)}\left(\left(\sinh K \frac{y^{*}}{2}+K^{2} P_{1}^{2} \frac{v_{0}}{2} \cosh K_{\frac{y^{*}}{2}}\right) e^{-v_{0} \frac{y^{*}}{4}}-K P_{1}^{2} \frac{v_{0}}{2}\right)}{\left(K \cosh K+\frac{1}{2} v_{0} \sinh K\right)\left(1-K^{2}\left(P_{1}^{2} \frac{v_{0}}{2}\right)^{2}\right)}+
$$$$
\frac{e^{P_{1}^{2}\left(t_{\max }-t^{*}\right)}\left(\frac{\cos t^{*}}{P_{1}^{2}}-\sin t^{*}\right)}{2 \operatorname{acosh} a P_{1}^{2}\left(1-\frac{1}{\left(P_{1}^{2}\right)^{2}}\right)}\left(\frac{1-e^{a \frac{y^{*}}{2}}}{1+P_{1}^{2}+a C}+\frac{e^{-a \frac{y^{*}}{2}}-1}{1+P_{1}^{2}-a C}\right)-\theta_{0} t^{* 2} \frac{y^{*}}{4}+
$$$$
\left(\theta_{0} C \frac{y^{* 2}}{8}+\operatorname{Pr} \theta_{0} \frac{y^{* 3}}{48}\right) t^{*}+\frac{e^{C}}{1-e^{C}}\left(t^{* 2} \frac{y^{*}}{4}-C \frac{y^{* 2}}{8} t^{*}-\right.
$$$$
\left.\left.\operatorname{Pr} \frac{y^{* 3}}{48} t^{*}\right)\right]
$$ 


\subsection{Dimensionless mass transfer}

The mass transfer is given by Equation (39) below as,

$m^{*}\left(y^{*}, t^{*}\right)=\rho_{0} Q^{*}\left(y^{*}\right)$.

By plugging Equation (38) in (39), one obtain the mass transfer as,

$$
\begin{aligned}
& m^{*}\left(y^{*}, t^{*}\right)=A^{*} c_{d} \rho_{0}\left[\frac { 1 } { c ^ { 2 } ( 1 - e ^ { - C } ) ( 1 - e ^ { v 0 } ) } \left(( 1 + \frac { P r } { 1 - P r } ) \left(\left(e^{-C}-\right.\right.\right.\right. \\
& \left.e^{v_{0}}\right) \frac{y^{*}}{2}-\left(e^{-C}-1\right) \frac{e^{v_{0} \frac{y^{*}}{2}}}{v_{0}}+\left(1-e^{v_{0}}\right) \frac{e^{-C \frac{y^{*}}{2}}}{C}+\frac{\left(e^{-C}-1\right)}{v_{0}}- \\
& \left.\frac{\left(1-e^{v_{0}}\right)}{C}\right)+\left(1-\theta_{0}\left(1-e^{-C}\right)\right)\left(-\operatorname{Pr}\left(v_{0}+\left(1-e^{v_{0}}\right)\right) \frac{y^{*}}{2}+\right. \\
& \left.\left.\operatorname{Pr} e^{v_{0} \frac{y^{*}}{2}}+\left(1-e^{v_{0}}\right)\left(\operatorname{Pr} \frac{y^{*}}{2}-C \frac{y^{* 2}}{8}\right)-P r\right)\right) t^{*}+ \\
& \frac{U_{0} \frac{v_{0}}{2} e^{P{ }_{1}^{2}\left(\left(t_{\max }-t^{*}\right)+\frac{1}{2} v_{0}\right)}\left(\left(\sinh K \frac{y^{*}}{2}+K^{2} P_{1}^{2} \frac{v_{0}}{2} \cosh K \frac{y^{*}}{2}\right) e^{-v_{0} \frac{y^{*}}{4}}-K P_{1}^{2} \frac{v_{0}}{2}\right)}{\left(K \cosh K+\frac{1}{2} v_{0} \sinh K\right)\left(1-K^{2}\left(P_{1}^{2} \frac{v_{0}}{2}\right)^{2}\right)}+ \\
& \frac{e^{P_{1}^{2}\left(t_{\max }-t^{*}\right)\left(\frac{\cos t^{*}}{P_{1}^{2}}-\sin t^{*}\right)}}{2 \operatorname{acosha} P_{1}^{2}\left(1-\frac{1}{\left(P_{1}^{2}\right)^{2}}\right)}\left(\frac{1-e^{a \frac{y^{*}}{2}}}{1+P_{1}^{2}+a C}+\frac{e^{-a \frac{y^{*}}{2}}-1}{1+P_{1}^{2}-a C}\right)-\theta_{0} t^{* 2} \frac{y^{*}}{4}+ \\
& \left(\theta_{0} C \frac{y^{* 2}}{8}+\operatorname{Pr} \theta_{0} \frac{y^{* 3}}{48}\right) t^{*}+\frac{e^{C}}{1-e^{C}}\left(t^{* 2} \frac{y^{*}}{4}-C \frac{y^{* 2}}{8} t^{*}-\right. \\
& \left.\operatorname{Pr} \frac{y^{* 3}}{48} t^{*}\right)
\end{aligned}
$$

\section{ASYMPTOTIC BEHAVIOR AND DISCUSSION OF THE RESULTS}

In this section the main features of the solutions found in the previous section (4.0) will be discussed. This is done in order to see the effect of changes of effective thermal coefficient $\theta_{0}$ to the overall distributions, while keeping other operating conditions and parameters fixed, and ascertain the best one for optimal natural ventilation.

A Physical interpretation of dimensionless temperature profiles for three incremental values of $\theta_{0}=(0.01,0.03,0.05)$ is presented in Figure 4, 5, and 6. In which in Figure 4 as $t^{*}$ increase the corresponding $\dot{\theta}^{*}\left(y^{*}, t^{*}\right)$ increases with $\theta_{0}=0.01$. In Figure 5 as $t^{*}$ increase the corresponding $\dot{\theta}^{*}\left(y^{*}, t^{*}\right)$ increases with $\theta_{0}=0.03$. And in Figure 6 as $t^{*}$ increase the corresponding $\dot{\theta}^{*}\left(y^{*}, t^{*}\right)$ also increases with $\theta_{0}=0.05$.The obvious features to be observed is that, all the lines of flow for temperature profiles across the openings are linearly distributed. Therefore, it is found that the best value of $\dot{\theta}^{*}\left(y^{*}, t^{*}\right)$ for optimal natural ventilation is when $\theta_{0}=0.01$ and $t^{*}=t_{\max }=1.0$.

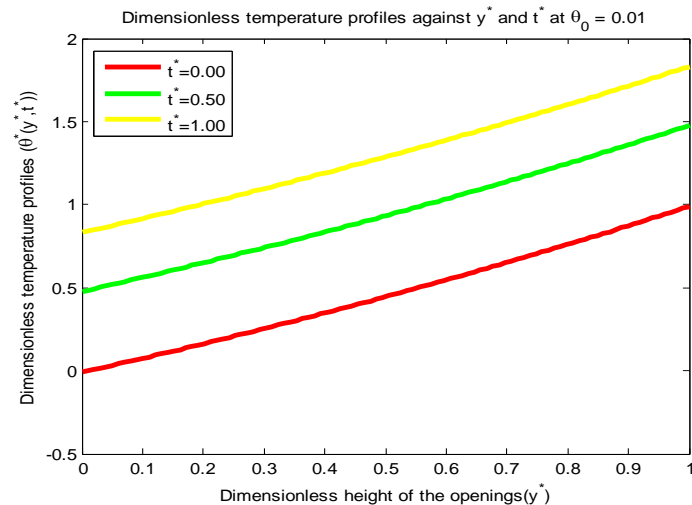

Figure 4: Dimensionless temperature profiles $\dot{\theta}^{*}$ versus $\boldsymbol{y}^{*}$ and $t^{*}$ at $\theta_{0}=0.01$.

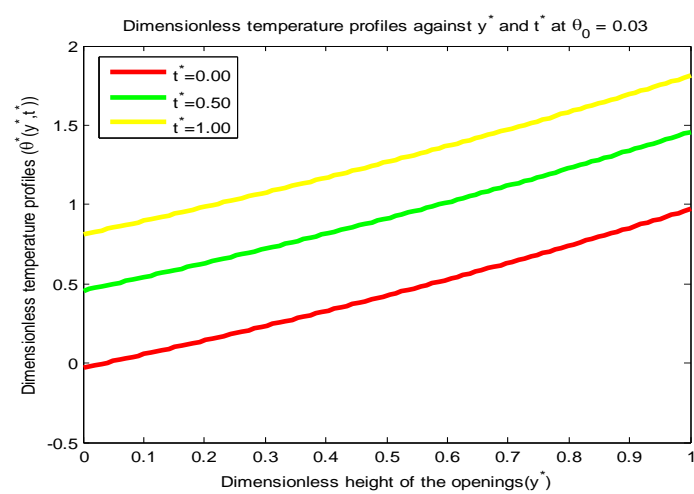

Figure 5: Dimensionless temperature profiles $\dot{\theta}^{*}$ versus $\boldsymbol{y}^{*}$ and $t^{*}$ at $\theta_{0}=0.03$.

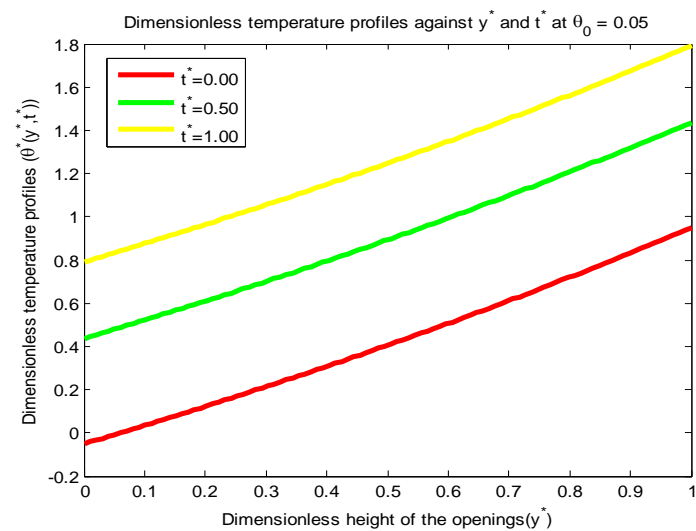

Figure 6: Dimensionless temperature profiles $\dot{\boldsymbol{\theta}}^{*}$ versus $\boldsymbol{y}^{*}$ and $\boldsymbol{t}^{*}$ at $\theta_{0}=0.05$

A Physical interpretation of dimensionless velocity profiles for three incremental values of $\theta_{0}=(0.01,0.03,0.05)$ is presented in Figure 7, 8, and 9. In which in Figure 7 as $t^{*}$ increase the corresponding $U^{*}\left(y^{*}, t^{*}\right)$ increases with $\theta_{0}=$ 0.01 . In Figure 8 as $t^{*}$ increase the corresponding $U^{*}\left(y^{*}, t^{*}\right)$ increases with $\theta_{0}=0.03$. And in Figure 9 as $t^{*}$ increase the corresponding $U^{*}\left(y^{*}, t^{*}\right)$ also increases with $\theta_{0}=0.05$. The obvious features to be observed is that, as $t^{*}$ increases the line of flow for velocity profiles across the openings also increases. Therefore, it is found that the best value of $U^{*}\left(y^{*}, t^{*}\right)$ for optimal natural ventilation is when $\theta_{0}=0.01$. and $t^{*}=t_{\max }=1.0$. 


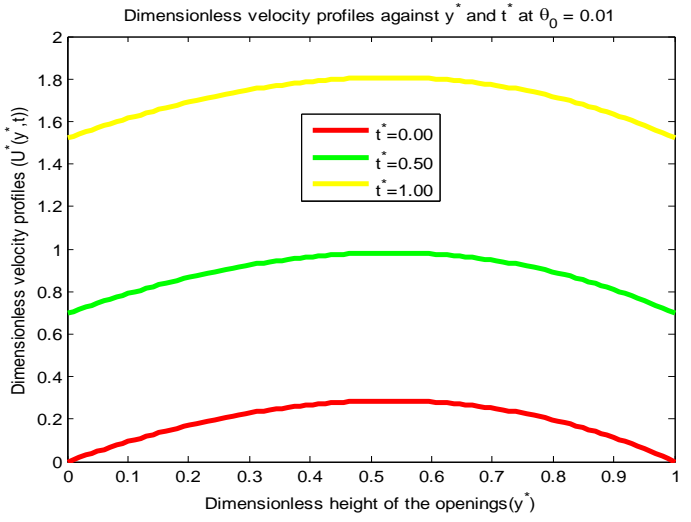

Figure 7: Transient Dimensionless velocity profiles $U^{*}$ versus $y^{*}$ and $t^{*}$ at $\theta_{0}=0.01$.

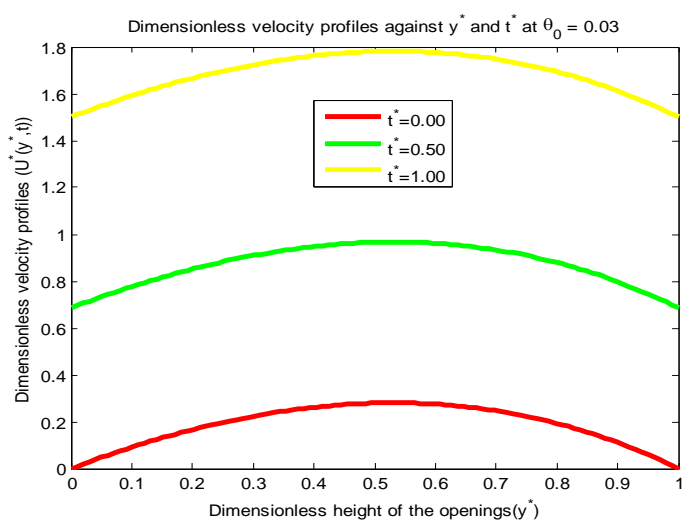

Figure 8: Transient Dimensionless velocity profiles $\boldsymbol{U}^{*}$ versus $y^{*}$ and $t^{*}$ at $\theta_{0}=0.03$.

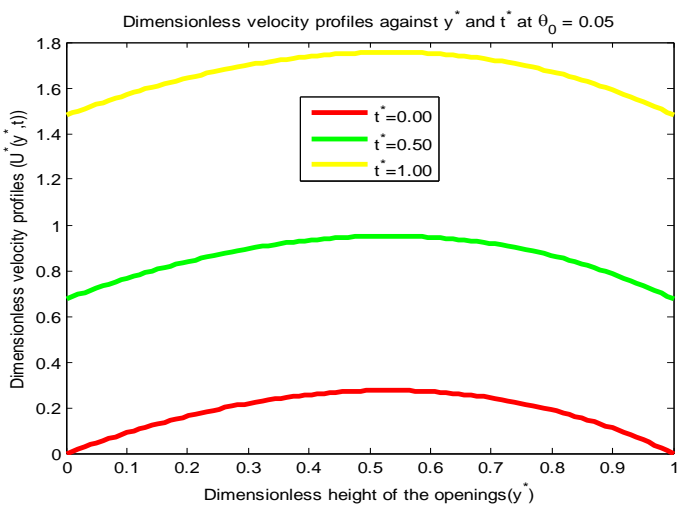

Figure 9: Transient Dimensionless velocity profiles $U^{*}$ versus $y^{*}$ and $t^{*}$ at $\theta_{0}=0.05$.

A Physical interpretation of dimensionless volumetric airflow for three incremental values of $\theta_{0}=(0.01,0.03,0.05)$ is presented in Figure 10, 11, and 12. In which in Figure 10 as $t^{*}$ increase the corresponding $Q^{*}\left(y^{*}, t^{*}\right)$ increases with $\theta_{0}=$ 0.01 . In Figure 11 as $t^{*}$ increase the corresponding $Q^{*}\left(y^{*}, t^{*}\right)$ increases with $\theta_{0}=0.03$. And in Figure 12 as $t^{*}$ increase the corresponding $Q^{*}\left(y^{*}, t^{*}\right)$ also increases with $\theta_{0}=0.05$. The obvious features to be observed is that, as $t^{*}$ increases the volumetric airflow also increases. Therefore, it is found that the best value of $Q^{*}\left(y^{*}, t^{*}\right)$ for optimal natural ventilation is when $\theta_{0}=0.01$ and $t^{*}=t_{\max }=1.0$.

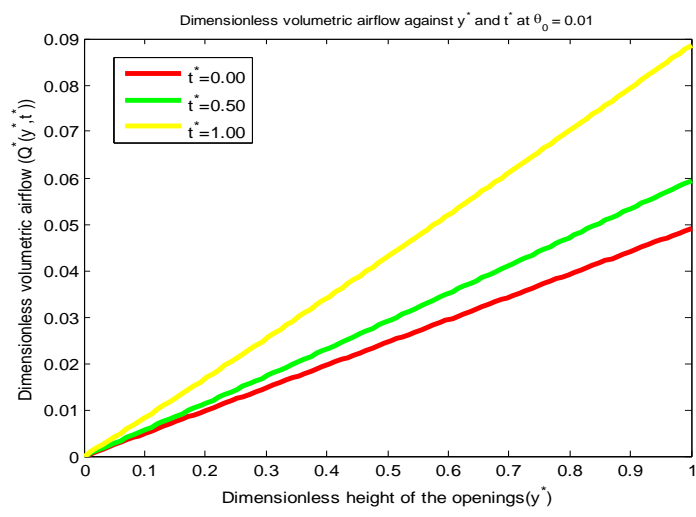

Figure 10: Transient Dimensionless volumetric airflow $Q^{*}$ versus $y^{*}$ and $t^{*}$ at $\theta_{0}=0.01$.

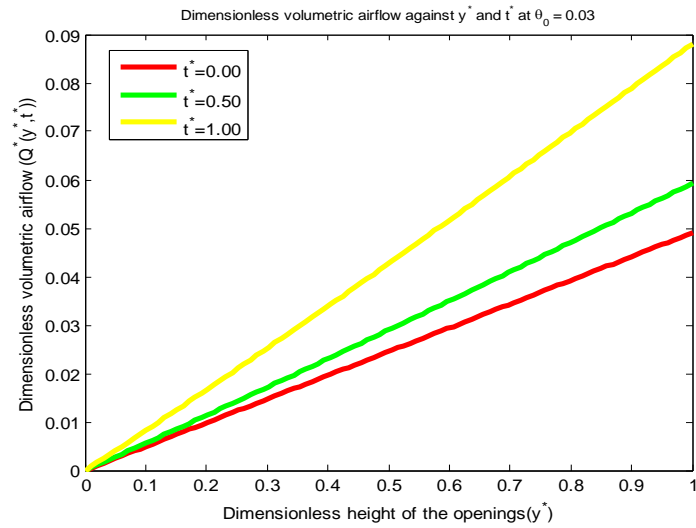

Figure 11: Transient Dimensionless volumetric airflow $Q^{*}$ versus $y^{*}$ and $t^{*}$ at $\theta_{0}=0.03$.

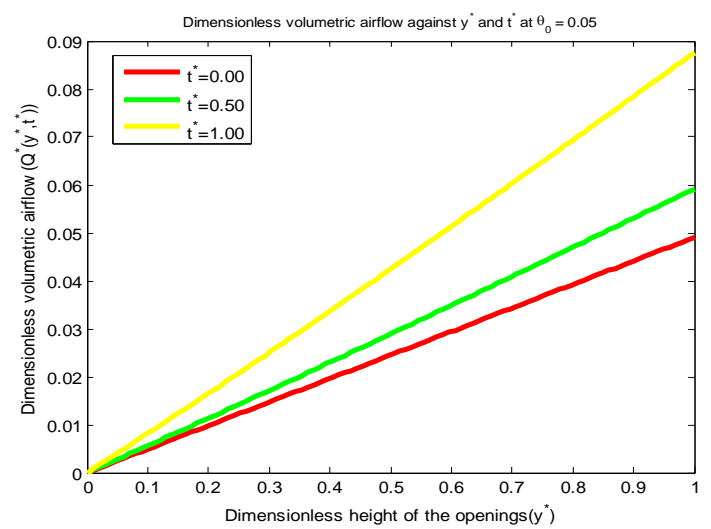

Figure 12: Transient Dimensionless volumetric airflow $Q^{*}$ versus $\boldsymbol{y}^{*}$ and $\boldsymbol{t}^{*}$ at $\boldsymbol{\theta}_{0}=\mathbf{0 . 0 5}$

A Physical interpretation of dimensionless mass transfer for three incremental values of $\theta_{0}=(0.10,0.30,0.50)$ is presented in Figure 13, 14, and 15. In which in Figure 13 as $t^{*}$ increase the corresponding $m^{*}\left(y^{*}, t^{*}\right)$ increases with $\theta_{0}=$ 0.01 . In Figure 14 as $t^{*}$ increase the corresponding $m^{*}\left(y^{*}, t^{*}\right)$ increases with $\theta_{0}=0.03$. And in Figure 15 as $t^{*}$ increase the corresponding $m^{*}\left(y^{*}, t^{*}\right)$ also increases with $\theta_{0}=0.05$. The obvious features to be observed is that, as $t^{*}$ increases the mass transfer also increases. Therefore, it is found that the best value of $m^{*}\left(y^{*}, t^{*}\right)$ for optimal natural ventilation is when $\theta_{0}=0.01$ and $t^{*}=t_{\max }=1.0$. 


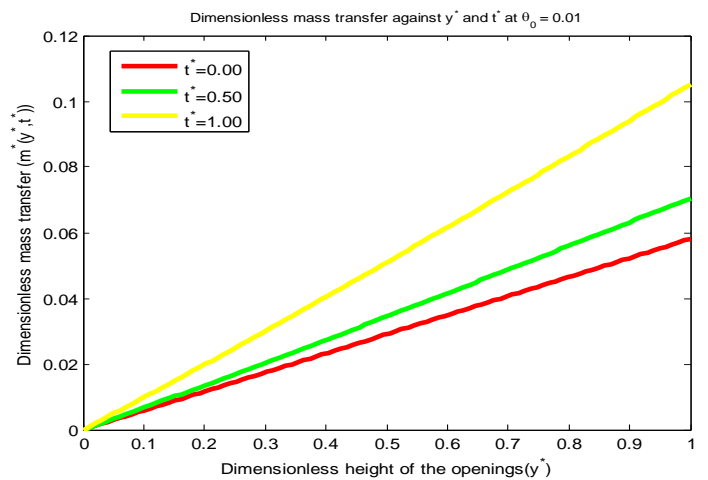

Figure 13: Transient Dimensionless mass transfer $\boldsymbol{m}^{*}$ versus $y^{*}$ and $t^{*}$ at $\theta_{0}=0.01$.

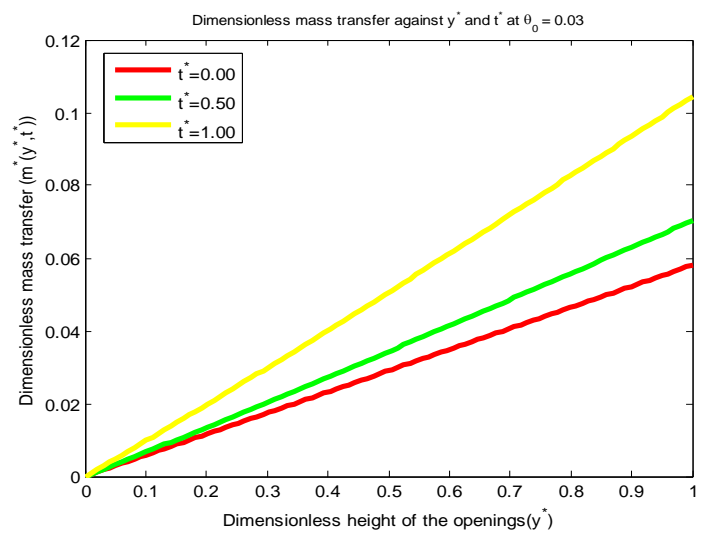

Figure 14: Transient Dimensionless mass transfer $\boldsymbol{m}^{*}$ versus $y^{*}$ and $t^{*}$ at $\theta_{0}=0.01$

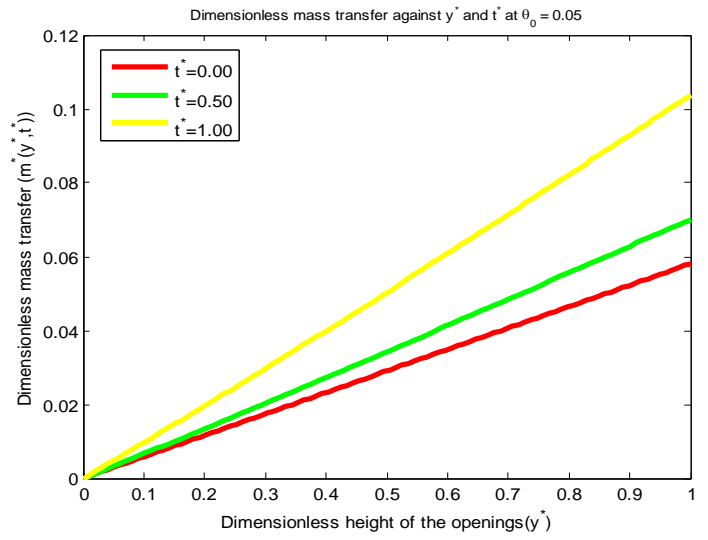

Figure 15: Transient Dimensionless mass transfer $\boldsymbol{m}^{*}$ versus $y^{*}$ and $t^{*}$ at $\theta_{0}=0.01$

\section{CONCLUSION}

A transient airflow process across three vertical vents induced by stack- driven effect inside un- stratified cross- ventilated rectangular building with an opposing flow in one of the upper opening was presented. Parameters such as, effective thermal coefficient and Prandtl number were also introduced, which were believed to have significant effects on natural ventilation process in buildings. Analytical techniques were employed to obtain the possible solutions of the model Equations, which predicts velocity- and temperature profiles together with volumetric airflow and mass-transfer. Various parameters on air flow process were used to see the effect of changes of effective thermal coefficient $\theta_{0}$ for different time intervals $\left(t^{*}\right)$ to the overall flow distributions, and ascertain the best one for optimal natural ventilation. Therefore, expected objectives in the paper are achieved.

The paper lead to the following conclusions:

1- A decrease in effective thermal coefficient $\theta_{0}$ results in an increase in temperature profiles $\dot{\theta}^{*}$ across the openings. The temperatures profiles $\dot{\theta}^{*}$ is more sensitive at lower values of effective thermal coefficient $\theta_{0}$. Therefore, the main features to be observed is that the temperature profiles $\dot{\theta}^{*}$ was within comfortable conditions for higher value of time intervals $t^{*}$.

2- A decrease in effective thermal coefficient $\theta_{0}$ results in an increase in velocity profiles $U^{*}$ across the openings. The velocity profiles $U^{*}$ is more sensitive at lower values of effective thermal coefficient $\theta_{0}$. Therefore, the main features to be observed is that the velocity profiles $U^{*}$ is higher in comparison to higher value of time intervals $t^{*}$.

3- A decrease in effective thermal coefficient $\theta_{0}$ result in an increase in volumetric airflow $Q^{*}$. The volumetric airflow $Q^{*}$ is more sensitive at lower values of effective thermal coefficient $\theta_{0}$. Therefore, the main features to be observed is that the volumetric airflow $Q^{*}$ is higher in comparison to higher value of time intervals $t^{*}$

4- An increase in effective thermal coefficient $\theta_{0}$, result in an increase in mass transfer $m^{*}$. The mass transfer $m^{*}$ is more sensitive at lower values of effective thermal coefficient $\theta_{0}$. Therefore, the main features to be observed in this research is that the mass transfer $m^{*}$ is higher in comparison to higher value of time intervals $t^{*}$.

5- The greater vertical distance between the openings, and the greater temperature difference between the inside and the outside, the stronger is the effect of the buoyancy.

The model is only valid for cross- ventilated building with three openings at the same height. Lastly, the research findings will help in developing a better understanding of natural ventilation process and help researchers to gain more insights into the phenomenon and therefore come up with more models.

\section{ACKNOWLEDGMENTS}

One of the author's is grateful, to authorities of KUST- Wudil for granting him leave to conduct the research.

\section{BIBLIOGRAPHY}

\subsection{Nomenclature}

$C_{1}, C_{2}, C_{3}, C_{4}$ Coefficients;

$K_{1}, K_{2}, a, K \quad$ Arbitrary constants;

$P_{1} \quad$ Separation constants;

$A^{*} \quad$ Total area of the openings;

$x_{w} \quad$ Width of the openings;

$y \quad$ Dimensional height of the openings;

$y^{*} \quad$ Dimensionless height of the openings;

$c_{d} \quad$ Discharge coefficient; 
$t^{*} \quad$ Dimensionless time intervals;

$t \quad$ Dimensional time intervals;

$q \quad$ Heat source;

$c_{p} \quad$ Specific heat capacity of air;

$t_{\max } \quad$ Maximum time;

$v_{0} \quad$ Constant velocity of the opposing flow;

$\rho_{0} \quad$ Constant density of the air;

$u, v \quad$ Velocity components along $x-, y$ - coordinate;

$U \quad$ Dimensional velocity profile;

$U^{*}\left(y^{*}, t^{*}\right)$ Dimensionless velocity profiles;

$U_{S}^{*}\left(y^{*}\right) \quad$ Dimensionless steady velocity profiles;

$U_{u}^{*}\left(y^{*}, t^{*}\right)$ Dimensionless unsteady velocity profiles;

$m^{*}\left(y^{*}, t^{*}\right) \quad$ Dimensionless mass - transfer;

$Q^{*}\left(y^{*}, t^{*}\right) \quad$ Dimensionless volumetric airflow;

$U_{C}\left(y^{*}\right), U_{C}\left(y^{*}, t^{*}\right) \quad$ Complimentary solutions;

$U_{p}\left(y^{*}\right), U_{p}\left(y^{*}, t^{*}\right) \quad$ Particular solutions;

$\frac{y^{*}}{2} \quad$ Neutral height;

$s \quad$ Dummy variable;

$L \quad$ Length scale of the height of the opening;

\subsubsection{Greek Symbols}

$\alpha \quad$ Thermal diffusivity;

$v \quad$ Kinematic viscosity;

$\kappa \quad$ Thermal conductivity;

$\mu \quad$ Dynamic viscosity;

$\Phi_{v} \quad$ Viscous dissipation;

$\beta \quad$ Thermal expansion coefficient;

$\theta_{0} \quad$ Effective thermal coefficient;

$\Delta \dot{\theta} \quad$ Dimensional change of air temperature;

$\dot{\theta}_{s}^{*}\left(y^{*}\right) \quad$ Dimensionless steady temperature profiles;

$\dot{\theta}_{u}^{*}\left(y^{*}, t^{*}\right)$ Dimensionless unsteady temperature profiles;

$\dot{\theta} \quad$ Dimensional temperature of air;

$\dot{\theta}^{*}\left(y^{*}, t^{*}\right)$ Dimensionless temperature profiles;

$\rho_{0} \quad$ Uniform interior air density;

\subsubsection{Dimensionless parameter}

$\mathrm{Pr} \quad$ Prandtl number;

\subsubsection{Subscripts}

w Width;

\section{REFERENCES}

[1] M.Santamouris, A. Argiriou, D. Asimakopoulos, N. Klitsikas, A. Dounis 1995. Heat and Mass- transfer through large openings by natural convection. Energy and Buildings 23.

[2] Liddament MW. 1991. A review of building air flow simulation. Tech. Note AIVC 33.

[3] Tong Yang, 2004. CFD and Field Testing of a naturally ventilated Full-scale Building. Doctoral Thesis. University of Nottingham.

[4] W. G. Brown and K. R. Salvason 1962. Natural convection through rectangular opening in partition-I. Int. J. Heat-Mass Transfer 5, 859- 868.

[5] Yuguo Li, Angelo Delsante, Je Symons 2000. Prediction of natural ventilation in buildings with large openings. Builing and Environment 35, 191- 206.

[6] Mostafa Rahimi and Imam Arianmehr 2009. The effect of a vertical vent on single-sided displacement ventilation. Department of Mech. Eng. University of Mohaghegh Ardabili, Iran.

[7] Murakami S. \& Kato S. 1989. Numerical and experimental study on room air flow- 3-D predictions using the $\mathrm{K}-\varepsilon$ turbulence model. Build. Environ. 24. 85- 97.

[8] C.L. Chow 2010. Air flow rate across vertical opening induced by room heat sources. International Journal on Architectural Science Volume 8, Number 1.11- 16.

[9] Liang Chung James Lo, 2012. Predicting wind driven cross ventilation in buildings with small openings. Doctoral thesis. University of Texas U. S. A.

[10] Andrew Acred, Gary R. Hunt 2014. A simplified mathematical approach for modelling stack ventilation in multi-compartment buildings. Builing and Environment $71,121-130$.

[11] A. L. Muhammad and A. B. Baffa 2015. Airflow process across vertical vents induced by stack- driven effect with an opposing flow in one of the upper openings. International Journal of Computer Application (IJCA), Volume 123(1). 1- 8

[12] A. L. Muhammad and A. B. Baffa and M. Z. Ringim 2016. Investigation of stack- driven airflow through rectangular cross- ventilated building with two openings using analytic technique. International Journal of Computer Application (IJCA), Volume 141(6). 5- 11. 